\title{
ZERO-DIMENSIONAL COMPACT ASSOCIATIVE DISTRIBUTIVE UNIVERSAL ALGEBRAS ${ }^{1}$
}

\author{
TAE HO CHOE
}

\begin{abstract}
We consider the question under which conditions a zero-dimensional compact universal algebra $\mathfrak{A}$ of finite type is profinite in the sense that the intersection of all closed congruences on $\mathfrak{A}$ with finite quotients is trivial. This is known ([2], [9]) to be the case for typical algebras such as groups, semigroups, Boolean lattices (or distributive lattices) and associative rings, but not in general. In this paper we show that if the underlying algebra $r \vec{i}$ the $\mathfrak{U}$ has generalized associativity and distributivity (see definitions in $\S 1$ ), then $\mathfrak{U}$ is always profinite. It then follows directly from [2] that the two categories of all residually finite associative, distributive universal algebras of the same finite type and of all zerodimensional compact ones are in adjoint situation. From this it is shown that all projectives in the latter category are completely characterized in terms of free algebras in the former category.
\end{abstract}

1. Terminologies and definitions. In this paper, terminologies and definitions mainly follow those in [5] for universal algebra, those in [8] for category theory and those in [2] for the others. Throughout this paper all universal algebras will be of finite type, i.e., by a $\tau$-algebra we mean a pair $\left(A,\left(f_{i}\right)_{i \in I}\right)$, where $A$ is a set, $\left(f_{i}\right)_{i \in I}$ a family of operations: maps $f_{i}$ of $A^{m_{i}}$ into $A, m_{i}$ the arity of the $f_{i}$ that is a nonnegative integer and $I$ is a finite ordered set $\{1,2, \cdots, t\}$. And we say that it is of arity type $\tau=\left(m_{1}, m_{2}, \cdots, m_{t}\right)$.

By a topological $\tau$-algebra we mean a triple $\left(A,\left(f_{i}\right)_{i \in I}, \mathscr{T}\right)$, where $\left(A,\left(f_{i}\right)_{i \in I}\right)$ is a $\tau$-algebra and $\mathscr{T}$ is a Hausdorff topology on $A$ under which each $f_{i}$ is a continuous map of the product space $\left(A^{m_{i}}, \mathscr{T}^{m_{i}}\right)$ into $(A, \mathscr{T})$.

Let $\mathfrak{U}$ be a $\tau$-algebra $\left(A,\left(f_{i}\right)_{i \in . V},\left(u_{p}\right)_{p \in M},\left(n_{r}\right)_{r \in L}\right)$, where $\left(f_{i}\right)_{i \in N}$ is the family of all operations with the arity $m_{i} \geqq 2,\left(u_{p}\right)_{p \in M}$ the family of all

Received by the editors January 31, 1973.

AMS (MOS) subject classifications (1970). Primary 54H10, 08A05; Secondary $22 \mathrm{~A} 30$.

Key words and phrases. Universal algebra, compact topological universal algebra, free algebra, projectivity.

${ }^{1}$ This research was supported by N.R.C. Grant A4809, Canada. 
unary operations $\left(m_{p}=1\right)$ and $\left(n_{r}\right)_{r \in L}$ all nullary ones $\left(m_{r}=0\right)$, and $N=$ $\{1,2, \cdots, n\}, M=\{n+1, \cdots, m\}$ and $L=\{m+1, \cdots, t\} . \mathscr{A}$ is said to be associative if for each $f_{i}(i \in N)$

$$
\begin{aligned}
f_{i}\left(f _ { i } \left(a_{1},\right.\right. & \left.\left.a_{2}, \cdots, a_{m_{i}}\right), a_{m_{i}+1}, \cdots, a_{2 m_{i}-1}\right) \\
& =f_{i}\left(a_{1}, f_{i}\left(a_{2}, \cdots, a_{m_{i}+1}\right), a_{m_{i}+2}, \cdots, a_{2 m_{i}-1}\right) \cdots \\
& =f_{i}\left(a_{1}, \cdots, f_{i}\left(a_{m_{i}}, \cdots, a_{2 m_{i}-1}\right)\right)
\end{aligned}
$$

for all $a_{k} \in A, k=1,2, \cdots, 2 m_{i}-1$, and for each $u_{p}$, there exist two integers $n(p) \geqq 0$ and $k(p) \geqq 1$ such that

for all $a \in A$.

$$
u_{p}^{n(p)+k(p)}(a)=u_{p}^{n(p)}(a)
$$

$\mathfrak{A}$ is said to be distributive if the following (i), (ii) and (iii) (or (i), (ii)' and (iii)) are satisfied:

(i) For each pair of $f_{i}$ and $f_{j}$ with $i<j$ in $N$,

$$
\begin{aligned}
& f_{j}\left(a_{1}, \cdots, a_{k-1}, f_{i}\left(b_{1}, \cdots, b_{m_{i}}\right), a_{k+1}, \cdots, a_{m_{j}}\right) \\
& =f_{i}\left(f_{j}\left(a_{1}, \cdots, a_{k-1}, b_{1}, a_{k+1}, \cdots, a_{m_{j}}\right), \cdots,\right. \\
& \left.f_{j}\left(a_{1}, \cdots, a_{k-1}, b_{m_{i}}, a_{k+1}, \cdots, a_{m}\right)\right)
\end{aligned}
$$

for all $a_{m}$ and $b_{n} \in A$ and all $k\left(k=1,2, \cdots, m_{j}\right)$.

(ii) For each $f_{i}$ and each $u_{p}$, either

or

$$
u_{p}\left(f_{i}\left(a_{1}, \cdots, a_{m_{i}}\right)\right)=f_{i}\left(u_{p}\left(a_{m_{i}}\right), \cdots, u_{\nu}\left(a_{1}\right)\right)
$$

$$
u_{\nu}\left(f_{i}\left(a_{1}, \cdots, a_{m_{i}}\right)\right)=f_{i}\left(a_{1}, \cdots, a_{k-1}, u_{p}\left(a_{k}\right), a_{k+1}, \cdots, a_{m_{i}}\right)
$$

for some $k\left(k=1,2, \cdots, m_{i}\right)$.

(ii)' For each $f_{i}$ and each $u_{p}$

$$
u_{p}\left(f_{i}\left(a_{1}, \cdots, a_{m_{i}}\right)\right)=f_{j}\left(u_{p}\left(a_{1}\right), \cdots, u_{p}\left(a_{m_{i}}\right)\right)
$$

for some $f_{j}$ with $m_{i}=m_{j}$.

(iii) For each pair of $u_{p}$ and $u_{q}, u_{q}\left(u_{p}(a)\right)=u_{p}\left(u_{q}(a)\right)$ for all $a \in A$.

Obvious classes of this type of algebras are, for example, sets with trivial operations, semigroups, distributive lattices, groups, rings, and lattice-ordered (semi) groups. Boolean lattices are of type (i), (ii)', and (iii). For a ring, $f_{1}$ is the addition, $f_{2}$ the multiplication, $u_{3}$ the unary operation of the inverse of $f_{1}$ and $N$ is the ordered set $\{1,2\}$.

Let $\mathfrak{U}=\left(A,\left(f_{i}\right)_{i \in I}\right)$ be a $\tau$-algebra. By a residually finite congruence relation $\theta$ we mean a congruence $\theta$ such that the cardinal $|\mathfrak{A} / \theta|$ of the quotient algebra of $\mathfrak{U}$ by $\theta$ is finite. An algebra $\mathfrak{U}$ is said to be residually finite [1] if the intersection of all residually finite congruences of $\mathfrak{A}$ is 
trivial, namely, the diagonal $\Delta_{A}$ of $A$. For example, every set with trivial operations, every distributive lattice and every Boolean lattice are always residually finite.

2. Zero-dimensional compact algebras. Let $\mathscr{A}$ be an equational class of associative and distributive $\tau$-algebras. Let $K \mathscr{A}\left(K_{0} \mathscr{A}\right)$ be the class of all topological $\tau$-algebras whose underlying spaces are compact (respectively, compact zero-dimensional) and whose underlying $\tau$ algebras belong to $\mathscr{A}$, and $F \mathscr{A}$ the class of all finite $\tau$-algebras belonging to $\mathscr{A}$. If each algebra in $F \mathscr{A}$ is given the discrete topology of its underlying set, then we have $F \mathscr{A} \subset K_{0} \mathscr{A} \subset K \mathscr{A}$. A closed congruence $\theta$ on $\mathfrak{U} \in K \mathscr{A}$ is said to have finite quotient if the quotient $\mathfrak{U} / \theta$ belongs to $F \mathscr{A}$. If the intersection of all closed congruences on $\mathfrak{A}$ with finite quotient is trivial then $\mathfrak{A}$ is called a profinite $\tau$-algebra. Let Pro $F \mathscr{A}$ be the class of all profinite $\tau$-algebras in $K \mathscr{A}$. It is known that Pro $F \mathscr{A} \subset K_{0} \mathscr{A}$. Regarding the identity Pro $F \mathscr{A}=K_{0} \mathscr{A}$, we know that groups, semigroups, distributive lattices and associative rings are always satisfied; on the other hand, there is the following counterexample [2]: the Stone-Cech compactification $\beta N$ of the set of all integers $N$ together with the continuous extension of the successor function: $n \rightarrow n+1$ is a zero-dimensional compact algebra, of type (1), but not profinite since it has only countably many open-closed congruences and $\beta N$ is not metrizable. Now we show in the following theorem that Pro $F \mathscr{A}=K_{0} \mathscr{A}$ for suitable $\mathscr{A}$.

THEOREM 1. If $\mathfrak{U}$ is a zero-dimensional compact associative distributive $\tau$-algebra, where $\tau=\left(m_{1}, \cdots, m_{k}\right)$, then $\mathfrak{A}$ is profinite.

Before the proof of the theorem, we give several lemmas and notation. The following lemma is well known.

LEMMA 1. If $X$ is a zero-dimensional compact topological space and $G$ is an open subset in the product space $X \times X$ containing the diagonal $\Delta_{X}$ then there exists a closed and open equivalence relation $R$ contained in $G$.

To simplify we introduce the following notation:

For $\left.\mathfrak{U}=\left(A, f_{i}\right)_{i \in N},\left(u_{p}\right)_{p \in M},\left(n_{r}\right)_{r \in L}\right) \in K_{0} \mathscr{A}$ let $R$ be an equivalence relation on $A$.

Let $(a, b)$ be a pair of elements in $A$.

For an operation $f_{i}(i \in N)$

$$
\begin{array}{r}
f_{i}[a] R f_{i}[b] \text { iff } f_{i}\left(x_{1}, \cdots, x_{k-1}, a, x_{k+1}, \cdots, x_{m_{i}}\right) \\
\equiv f_{i}\left(x_{1}, \cdots, x_{k-1}, b, x_{k+1}, \cdots, x_{m_{i}}\right)(R)
\end{array}
$$


for all $x_{l} \in A\left(l=1,2, \cdots, k-1, k+1, \cdots, m_{i}\right)$ and all $k(k=1,2, \cdots$, $\left.m_{i}\right)$.

$$
f_{i} f_{j}[a] R f_{i} f_{j}[b] \text { iff } f_{i}\left[f_{j}[a]\right] R f_{i}\left[f_{j}[b]\right]
$$

namely,

$$
\begin{aligned}
f_{i}\left(x_{1}, \cdots, x_{k-1}, f_{j}\left(y_{1}, \cdots, y_{l-1}, a, y_{l+1}, \cdots, y_{m_{j}}\right), x_{k+1}, \cdots, x_{m_{i}}\right) \\
\quad \equiv f_{i}\left(x_{1}, \cdots, x_{k-1}, f_{j}\left(y_{1}, \cdots, b, \cdots, y_{m_{j}}\right), x_{k+1}, \cdots, x_{m_{i}}\right)(R)
\end{aligned}
$$

for all $x_{m}$ and $y_{h} \in A$, and all $k$ and $l\left(k=1,2, \cdots, m_{i}, l=1,2, \cdots\right.$, $\left.m_{j}\right) \cdots$.

(r) $f_{i} f_{j} \cdots f_{p}[a] R f_{i} f_{j} \cdots f_{p}[b]$

$$
\text { iff } f_{i}\left[f_{j} \cdots\left[f_{p}[a]\right] \cdots\right] R f_{i}\left[f_{j} \cdots\left[f_{p}[a]\right] \cdots\right] \text {. }
$$

LEMMA 2. If $R$ is an equivalence relation on $\mathfrak{U}=\left(A,\left(f_{i}\right)_{i \in N},\left(u_{p}\right)_{p \in M}\right.$, $\left.\left(n_{r}\right)_{r \in L}\right)$ then $f_{i} f_{i}[a] R f_{i} f_{i}[b]$ implies $f_{i} f_{i} f_{i}[a] R f_{i} f_{i} f_{i}[b]$ for each pair $(a, b)$ of elements in $A$.

Proof. Let $(a, b)$ satisfy $f_{i} f_{i}[a] R f_{i} f_{i}[b]$.

By the associative law, we have that, for each $j, k, l \in\left\{1,2, \cdots, m_{i}\right\}$,

$$
\begin{array}{r}
f_{i}\left(x_{1}, \cdots, x_{j}, f_{i}\left(y_{1}, \cdots, y_{k}, f_{i}\left(z_{1}, \cdots, z_{l}, a, \cdots, z_{m_{i}}\right) \cdots y_{m_{i}}\right) \cdots x_{m_{i}}\right) \\
=f_{i}\left(f_{i}\left(x_{1}, \cdots, x_{j}, y_{1}, \cdots, y_{k}, z_{1}, \cdots, z_{l}, a, \cdots\right), \cdots, f_{i}\left(z_{m_{i}}, \cdots\right), \cdots, x_{m_{i}}\right) \\
\text { if } j+k+l+1 \leqq m_{i}, \\
=f_{i}\left(f_{i}\left(f_{i}\left(x_{1}, \cdots, x_{j}, y_{1}, \cdots, y_{k}, z_{1}, \cdots\right) \cdots z_{l}, a, \cdots, z_{m_{i}}, \cdots, y_{m_{i}}, \cdots, x_{m_{i}}\right)\right. \\
\text { if } j+k+l+1>m_{i} .
\end{array}
$$

In either case, by hypothesis, the expression is $R$-equivalent to the corresponding expression replacing $a$ by $b$. Hence we have $f_{i} f_{i} f_{i}[a] R f_{i} f_{i} f_{i}[b]$.

LEMMA 3. With the same notation as in Lemma 2,

implies

$$
f_{j} \cdots f_{k} f_{i} f_{i} f_{l} \cdots f_{m}[a] R f_{j} \cdots f_{k} f_{i} f_{i} f_{l} \cdots f_{m}[b]
$$

$$
f_{j} \cdots f_{k} f_{i} f_{i} f_{i} f_{l} \cdots f_{m}[a] R f_{j} \cdots f_{k} f_{i} f_{i} f_{i} f_{l} \cdots f_{m}[b] .
$$

Proof. Let $a^{\prime}$ be an arbitrary expression in $f_{l} \cdots f_{m}[a]$ and $b^{\prime}$ its corresponding expression replacing $a$ by $b$ in $f_{l} \cdots f_{m}[b]$. By the same argument as in the proof of Lemma 2, it follows that $f_{j} \cdots f_{k} f_{i} f_{i} f_{i}\left[a^{\prime}\right] R$ $f_{j} \cdots f_{k} f_{i} f_{i} f_{i}\left[b^{\prime}\right]$. Hence we have the required result.

ProOF OF THEOREM 1. Let $\mathfrak{U}=\left(A,\left(f_{i}\right)_{i \in N},\left(u_{p}\right)_{p \in M},\left(n_{r}\right)_{r \in L}, \mathscr{T}\right)$, where $N=\{1,2, \cdots, n\}$ and $M=\{n+1, \cdots, m\}$. By Lemma $1, \mathfrak{A}$ has a closed and open equivalence relation $R$ such that $R \subset G$ for a given open subset $G$ in $(A \times A, \mathscr{T} \times \mathscr{T})$ containing $\Delta_{A}$. 
Let $\eta$ be the set of all pairs $(a, b) \in R$ satisfying the following $2 n$ conditions $\left(\mathrm{C}_{1}\right) \sim\left(\mathrm{C}_{2 n}\right)$ :

$\left(\mathrm{C}_{1}\right): f_{i}[a] R f_{i}[b]$ for $i=1,2, \cdots, n$,

$\left(\mathrm{C}_{2}\right): f_{i} f_{j}[a] R f_{i} f_{j}[b]$ for all $i$ and $j \in N$ with $i \leqq j, \cdots$

$\left(\mathrm{C}_{r}\right): f_{i} f_{j} \cdots f_{l}[a] R f_{i} f_{j} \cdots f_{l}[b]$ for all $i, j, \cdots, l \in N$ with $i \leqq$ $j \leqq \cdots \leqq l$, where the number of operations $f_{i}, \cdots, f_{l}$ is $r, \cdots$

$\left(\mathrm{C}_{2 n}\right): f_{1} f_{1} f_{2} f_{2} \cdots f_{n} f_{n}[a] R f_{1} f_{1} f_{2} f_{2} \cdots f_{n} f_{n}[b]$

where all indices $i, j, \cdots, l \in N$ in $\left(\mathrm{C}_{1}\right) \sim\left(\mathrm{C}_{2 n}\right)$ are allowed to repeat not more than twice. Then $\eta$ is a congruence on the algebra $\left(A,\left(f_{i}\right)_{i \in N}\right)$ without unary and nullary operations. In fact, since $\eta$ is clearly an equivalence relation on $A$, it suffices to show that for each pair $(a, b) \in \eta$, $f_{k}[a] \eta f_{k}[b]$ is satisfied for all $k \in N$ [5]. To this end, we show that the pair $\left(f_{k}[a], f_{k}[b]\right)$ satisfies $\left(\mathrm{C}_{1}\right) \sim\left(\mathrm{C}_{2 n}\right)$. Firstly, $f_{k}[a] R f_{k}[b]$ is true by $\left(C_{1}\right)$. To check the condition $\left(C_{1}\right)$ of the pair $\left(f_{k}[a], f_{k}[b]\right)$, we observe two cases:

(1) if $i \leqq k$ in $N$, then by $\left(\mathrm{C}_{2}\right) f_{i} f_{k}[a] R f_{i} f_{k}[b]$,

(2) if $k<i$ in $N$ then by the distributivity of $f_{k}$ and $f_{i}$, we may interchange $f_{i}$ and $f_{k}$ so that we can apply $\left(\mathrm{C}_{2}\right)$ to obtain $f_{i} f_{k}[a] R f_{i} f_{k}[b]$.

Now to check the condition $\left(\mathrm{C}_{r}\right)$, we have to consider several cases: (1) if $i \leqq \cdots \leqq l \leqq k$ and $k$ is not repeated more than twice, then by $\left(\mathrm{C}_{r+1}\right)$ we have directly $f_{i} f_{j} \cdots f_{l} f_{k}[a] R f_{i} f_{j} \cdots f_{l} f_{k}[b],(2)$ if $i \leqq \cdots \leqq l \leqq k$ and $k$ is repeated three times, then by Lemma 3 and $\left(C_{r}\right)$ we have the same result, (3) if $l=k$, then by the distributivity and Lemma 3 , we may arrange the indices $\{i, \cdots, l, k\}$ in the natural order and $k$ is not repeated more than twice so that one can apply $\left(\mathrm{C}_{r}\right)$ or $\left(\mathrm{C}_{r+1}\right)$ to obtain the same result. Finally, for $\left(C_{2 n}\right), k$ must be repeated three times. Therefore applying the distributivity first and Lemma 3 second we have $f_{1} f_{1} \cdots f_{n} f_{n} f_{k}[a] R$ $f_{1} f_{1} \cdots f_{n} f_{n} f_{k}[b]$ by $\left(\mathrm{C}_{2 n}\right)$. Hence $\eta$ is a congruence on $\left(A,\left(f_{i}\right)_{i \in N}\right)$.

Now we show further that $\eta$ is closed and open in $(A \times A, \mathscr{T} \times \mathscr{T})$. Let $(a, b) \in \eta$. Since $R$ is open and all $f_{i}$ are continuous, there exist open neighborhoods $U_{a}$ and $V_{b}$ of $a$ and $b$, respectively, such that $U_{a} \times V_{b} \subset \eta$. For each given expression in $\left(C_{r}\right)$ we can find open neighborhoods $U$ and $V$ of $a$ and $b$, respectively, such that every pair of elements in $U \times V$ satisfies the given expression. Since the number of all different expressions in $\left(\mathrm{C}_{r}\right)(r=1,2, \cdots, 2 n)$, is at most finite, the intersections $U_{a}$ and $V_{b}$ of all such neighborhoods $U$ of $a$ and $V$ of $b$, respectively, are still open, and $U_{a} \times V_{b} \subset \eta$. Thus $\eta$ is open. It is easy to see that $\eta$ is closed since $R$ is.

Now we construct a closed and open congruence $\theta$ on the same algebra $\mathfrak{A}$ such that $\theta \subset \eta$. Let $\theta$ be the set of all pairs $(a, b)$ in $\eta$ satisfying the following conditions:

$\left(D_{1}\right) \sim\left(D_{s-1}\right):$

$\left(\mathrm{D}_{r}\right): u_{p}\left(u_{q}\left(\cdots\left(u_{t}(a)\right) \cdots\right)\right) \equiv u_{p}\left(u_{q}\left(\cdots\left(u_{t}(b)\right) \cdots\right)\right)(\eta), r=1,2, \cdots, s-1$, 
where $s=(n(p)+k(p))+\cdots+(n(t)+k(t))$ and each index $p$ in $\left(\mathrm{D}_{r}\right)$, $r=1,2, \cdots, s-1$, may be repeated a number of times less than $n(p)+k(p)$. Then by the distributivities (ii) and (iii) (or (ii)' ${ }^{\prime}$ and (iii)), it is not difficult to see that $\theta$ is also a closed and open congruence on $\mathfrak{A}$, and $\theta \subset \eta \subset R \subset G$. Furthermore, $\theta$ is residually finite since $\theta$ is open and $\mathscr{T}$ is compact. On the other hand, the set $G$ is an arbitrary open subset in $(A \times A, \mathscr{T} \times \mathscr{T})$ containing $\Delta_{A}$, and $\mathscr{T}$ is Hausdorff. Thus the intersection of all closed and open congruences on $\mathfrak{U}$ is trivial. It follows that the $\mathfrak{U}=\left(A,\left(f_{i}\right)_{i \in N}\right.$, $\left.\left(u_{p}\right)_{D \in M},\left(n_{r}\right)_{r \in L}, \mathscr{T}\right)$ is profinite.

REMARK. The hypotheses of associativity and distributivity in Theorem 1 are not necessary, for example, profinite nondistributive lattice etc. However, it is clear by Birkhoff's Subdirect Representation Theorem that for an equational class $\mathscr{A}$ if each subdirectly irreducible $\tau$-algebra in $F \mathscr{A}$ is associative and distributive, then Pro $F \mathscr{A}=K_{0} \mathscr{A}$ iff all $\mathfrak{A}$ in $K_{0} \mathscr{A}$ are associative and distributive.

3. Projectives in $K_{0} \mathscr{A}$. Let $\mathscr{A}$ be an equational class of associative, distributive $\tau$-algebras, and let $\mathscr{A}_{0}$ be the class of all residually finite $\tau$-algebras in $\mathscr{A}$. Then $\mathscr{A}_{0}$ is evidently hereditary and productive in $\mathscr{A}$. Consequently, it has free algebras over $\mathscr{A}_{0}$ with any set as the free generating set. For $\mathfrak{A} \in \mathscr{A}_{0}$, the family of all congruences $\theta$ with $\mathfrak{x} / \theta \in F \mathscr{A}$ forms a basis of a separated uniformity (called the $F \mathscr{A}$-adic uniformity) on the underlying set $A$ of $\mathfrak{A}$ [2]. The completion of the resulting uniform space is called the $F \mathscr{A}$-adic completion of $A$, denoted by $P A$. Since Pro $F \mathscr{A}=K_{0} \mathscr{A}$ by Theorem 1, we have the following lemma from [2]:

LEMMA 4. The functor $P$ of $F \mathscr{A}$-adic completion is left adjoint to the underlying algebra functor $U: K_{0} \mathscr{A} \rightarrow \mathscr{A}_{0}$.

By a projective $\mathfrak{P}$ in $K_{0}$, we mean that for an onto morphism $f: \mathfrak{A} \rightarrow \mathfrak{B}$ and a morphism $g: \mathfrak{B} \rightarrow \mathfrak{B}$ in $K_{0} \mathscr{A}$, there exists $h: \mathfrak{P} \rightarrow \mathfrak{A}$ in $K_{0} \mathscr{A}$ such that $f h=g$.

THEOREM 2. $\mathfrak{P}$ is projective in $K_{0} \mathscr{A}$ iff $\mathfrak{P}$ is a retract of the $F \mathscr{A}$-adic completion of a free algebra over $\mathscr{A}_{0}$.

Proof. Suppose that $\mathfrak{P}$ is projective in $K_{0} \mathscr{A}$. Let $\mathscr{F}$ be the free algebra over $\mathscr{A}_{0}$ generated by the underlying set $S$ of $\mathfrak{P}$. Let $U \mathfrak{B}$ be the underlying algebra of $\mathfrak{P}$. Then we have an onto homomorphism $h: \mathscr{F} \rightarrow U \mathfrak{P}$ such that $\left.h\right|_{s}=1_{s}$. The map $h$, being uniformly continuous with respect to the $F \mathscr{A}$-adic uniformities, extends uniquely to an onto morphism $P h: P \mathscr{F} \rightarrow P(U \mathfrak{P})$ in $K_{0} \mathscr{A}$ [2]. Moreover, there exists an onto morphism $g: P(U \mathfrak{P}) \rightarrow \mathfrak{P}$, namely the back adjunction for $\mathfrak{P}$. Since $\mathfrak{P}$ is projective, 
for the composition $g(P h)$ and the identity $1_{\mathfrak{P}}$ of $\mathfrak{P}$, we have a morphism $\varphi: \mathfrak{P} \rightarrow P \mathscr{F}$ such that $g(P h) \varphi=1_{\mathfrak{P}}$. Thus $\mathfrak{P}$ is a retract of $P \mathscr{F}$. The converse is directly from Lemma 4 and the fact that left adjoint functor preserves projectivity provided that the right adjoint preserves the onto morphisms [7].

REMARK. For any equational class $\mathscr{A}$, Lemma 4 is still true if $K_{0} \mathscr{A}$ is replaced by Pro $F \mathscr{A}$ [2]. Thus Theorem 2 is, after all, true for any $\mathscr{A}$ with Pro $F \mathscr{A}$ in the place of $K_{0} \mathscr{A}$.

\section{REFERENCES}

1. L. W. Anderson and R. P. Hunter, On residual properties of certain semigroups, Contributions to Extension Theory of Topological Structures (Proc. Sympos., Berlin, 1967), Deutsch. Verlag Wissen., Berlin, 1969, pp. 15-19. MR 39 \#7017.

2. B. Banaschewski, On profinite universal algebras, Proc. Third Topological Conference, Prague, September, 1971.

3. - Projective covers in categories of topological spaces and topological algebras, General Topology and its Relations to Modern Analysis and Algebra, III (Proc. Conf., Kanpur, 1968), Academia, Prague, 1971, pp. 63-91. MR 44 \#1616.

4. N. Bourbaki, General topology. Part I. Hermann, Paris; Addison-Wesley, Reading Mass., 1966. MR 34 \#5044a.

5. G. Grätzer, Universal algebra, Van Nostrand, Princeton, N.J., 1968. MR 40 \#1320.

6. K. W. Gruenberg, Projective profinite groups, J. London Math. Soc. 42 (1967), 155-165. MR 35 \#260.

7. S. Mac Lane, Homology, Die Grundlehren der math. Wissenschaften, Band 114, Academic Press, New York; Springer-Verlag, Berlin, 1963. MR 28 \#122.

8. B. Mitchell, Theory of categories, Pure and Appl. Math., vol. 17, Academic Press, New York, 1965. MR 34 \#2647.

9. K. Numakura, Theorems on compact totally disconnected semigroups and lattices, Proc. Amer. Math. Soc. 8 (1957), 623-626. MR 19, 290.

Department of Mathematics, McMaster University, Hamilton, Ontario, CANADA 